\title{
Peculiar outbursts of a black hole X-ray transient, V4641 Sgr
}

\author{
M. Uemura, ${ }^{1} \dagger$ T. Kato, ${ }^{2}$ D. Nogami, ${ }^{2}$ A. Imada,${ }^{2}$ and R. Ishioka ${ }^{3}$ \\ ${ }^{1}$ Astrophysical Science Center, Hiroshima Univ., Kagamiyama, Higashi-Hiroshima, Japan \\ ${ }^{2}$ Department of Astronomy, Kyoto University, Kitashirakawa, Kyoto, Japan \\ ${ }^{3}$ National Astronomical Observatory of Japan, Mitaka, Japan \\ uemuram@hiroshima-u.ac.jp
}

\begin{abstract}
We have kept optical monitoring of a peculiar black hole X-ray binary, V4641 Sgr. Based on our observations, we show that its unprecedented activity can be divided into 5 phases. In this paper, we report their observational properties.
\end{abstract}

Keywords. Accretion, accretion disks - black hole physics - binaries: close - stars: individual (V4641 Sgr)

\section{Introduction}

V4641 Sgr is a black hole X-ray binary (BHXB), which has shown mysterious outbursts almost once a year (Uemura et al. 2002; Uemura et al. 2004a; Uemura et al. 2004b; Uemura et al. 2005). We pay attention to this object, not only because of its peculiar behaviour, but also because it provides us with a number of information about topics which have recently received much attention in BHXBs, such as the jet-disk interaction (e.g. Mirabel et al. 1998), the synchrotron emission in the optical and IR regions (e.g. Fender 2001), and rapid optical variations (e.g. Kanbach et al. 2001). We keep on monitoring V4641 Sgr since 1999, and observed 5 optical outbursts. Here, we summarize optical properties of V4641 Sgr outbursts.

\section{Result and Discussion}

Its outbursts are quite unique in the points of short durations and rapid time-evolutions. Due to these characteristics, the number of data during outbursts is so small that it had been difficult to give a unified description for them even phenomenologically. Based on our long monitoring, however, we have finally found common properties in outbursts in 1999, 2002, 2003, 2004, and 2005. Photometric observations were performed using 30$\mathrm{cm}$ class telescopes at Kyoto University, Universidad de Concepcion, and a number of observatories under collaborations by VSNET.

Whole light curves of the previous five outbursts are shown in the left panel of Fig. 1. The outburst duration is typically one week, while the object keeps active even after the main outbursts. The state of V4641 Sgr can be divided in 5 phases based on its optical variability; i) the initial rising phase, ii) the high variability phase, iii) the superEddington flare, iv) the post-outburst active phase, and v) the quiescence. During the phases ii) and iv), the object exhibits rapid optical variations with time-scales of a few seconds. No periodic or quasi-periodic signal has been found in these phases (Uemura et al. 2004a; Uemura et al. 2004b). While X-ray luminosities were low in the phases ii)

$\dagger$ Present address: Kagamiyama 1-3-1, Higashi-Hiroshima, Japan. 

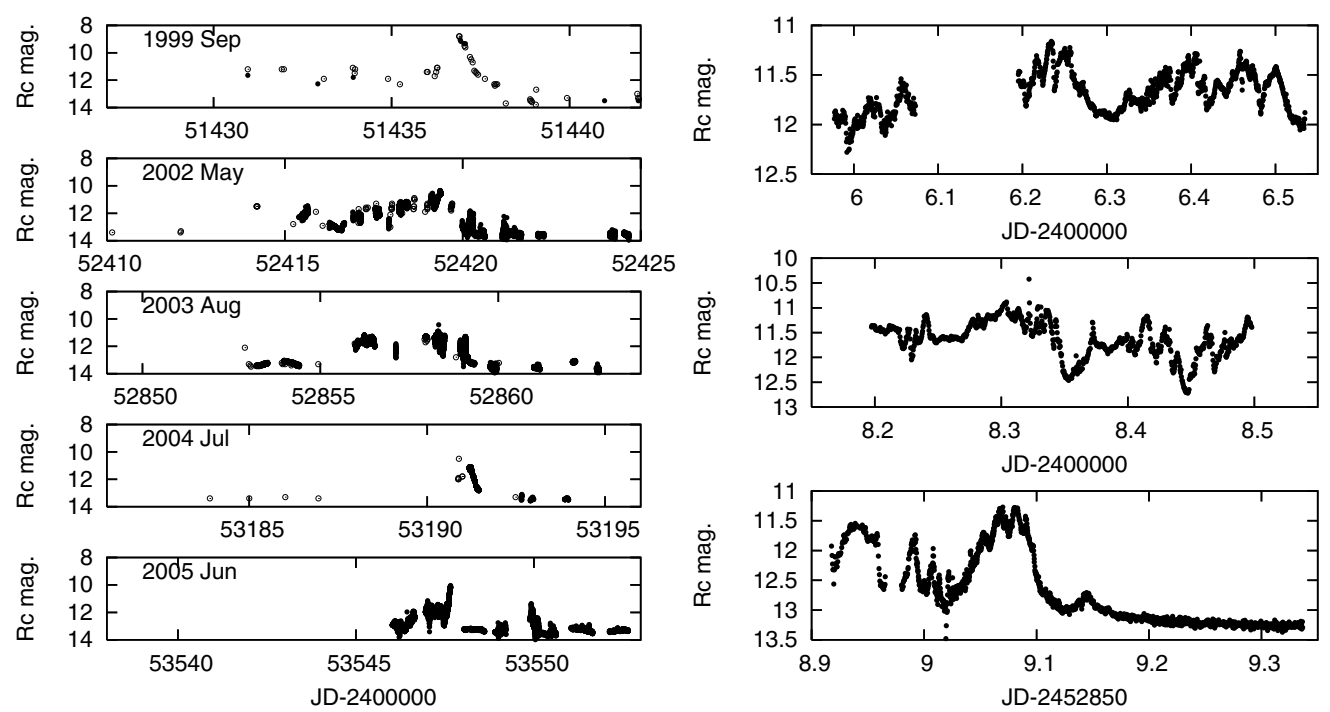

Figure 1. Left: 5 outbursts of V4641 Sgr. Right: Short-term variations during outbursts.

and iv) ( $L_{X} \sim 10^{35-36} \mathrm{erg} \mathrm{s}^{-1}$; Uemura et al. 2004b; Swank et al. 2005), it has reached super-Eddington levels during the final flares, at least in 1999 and 2005, and possibly in 2002 (Hjellming et al. 2000; Shimokawabe et al. 2005). Relativistic jets were associated to those flares (Hjellming et al. 2000).

Between the high variability phase and the super-Eddington flare, we may see a transition of accretion flows from the radiatively inefficient flow to the slim disk. We can expect a strong disk wind at the forming of the slim disk, which might have been observed as quite strong and broad emission lines observed during the final flare in 2004 (Lindstrom et al. 2005).

\section{Acknowledgements}

This research was partially supported by the Ministry of Education, Science, Sports and Culture, Grant-in-Aid for Young Scientists, 2006.

\section{References}

Fender, R. P. 2001, MNRAS, 322, 31

Hjellming, R. M. et al. 2000, ApJ, 544, 977

Kanbach, G., Straubmeier, C. \& Spruit, H. 2001, Nature, 414, 180

Lindstrom, C. et al. 2005, MNRAS, 363, 882

Mirabel, I. F. et al. 1998, A\&A, 330, L9

Shimokawabe, T. et al. 2005, Autumn Meeting of Astronomical Society of Japan

Swank, J. 2003, ATel, 303

Swank, J. 2005, ATel, 536

Uemura, M. et al. 2002, PASJ, 54, 95

Uemura, M. et al. 2004, PASJ, 56, 823

Uemura, M. et al. 2004, PASJ, 56, S61

Uemura, M. et al. 2005, IBVS, 5626 